\title{
Quantum-Phase-Field: From de Broglie-Bohm Double-Solution Program to Doublon Networks
}

https://doi.org/10.1515/zna-2019-0343

Received November 22, 2019; accepted December 11, 2019

\begin{abstract}
Different forms of linear and non-linear field equations, so-called 'phase-field' equations, are studied in relation to the de Broglie-Bohm double-solution program. This defines a framework in which elementary particles are described by localised non-linear wave solutions moving by the guidance of a pilot wave, defined by the solution of a Schrödinger-type equation. First, we consider the phase-field order parameter as the phase for the linear pilot wave, second as the pilot wave itself, and third as a moving soliton interpreted as a massive particle. In the last case, we introduce the equation for a superwave, the amplitude of which can be considered as a particle moving in accordance to the de Broglie-Bohm theory. Lax pairs for the coupled problems are constructed in order to discover possible non-linear equations that can describe the moving particle and to propose a framework for investigating coupled solutions. Finally, doublons in $1+1$ dimensions are constructed as self-similar solutions of a non-linear phase-field equation forming a finite space object. Vacuum quantum oscillations within the doublon determine the evolution of the coupled system. Applying a conservation constraint and using general symmetry considerations, the doublons are arranged as a network in $1+1+2$ dimensions, where nodes are interpreted as elementary particles. A canonical procedure is proposed to treat charge and electromagnetic exchange.
\end{abstract}

Keywords: Field Equation for Charged Particles; Lax Formalism of Coupled Wave Equations; Quantum Fluctuations; Wave Theory of Matter.

PACS numbers: 04.20.Cv; 04.50.Kd; 05.70.Fh

\footnotetext{
*Corresponding author: I. Steinbach, Interdisciplinary Centre for Advanced Materials Simulation, Ruhr-University Bochum, Universitaetsstrasse 150, 44801 Bochum, Germany, E-mail: ingo.steinbach@rub.de

J. Kundin: Interdisciplinary Centre for Advanced Materials Simulation, Ruhr-University Bochum, Universitaetsstrasse 150, 44801 Bochum, Germany, E-mail: julia.kundin@rub.de
}

\section{Introduction}

Since the emergence of quantum theory, physicists have tried to understand the physical world as a dual phenomenon of waves and particles. A very promising concept, but almost forgotten today, is the double-solution program of de Broglie and Bohm (dBB) [1-4]. The concept is based on two coupled wave equations. The so-called 'pilot wave' corresponds to the probability wave, $\psi$, in the Copenhagen interpretation of quantum mechanics and evolves according to a linear Schrödinger-type equation. This pilot wave couples to the $u$ wave (in de Broglie's notation [3]), which shall describe physical 'particles'. Doing this, it is possible to interpret e.g. the famous 'double-slit' experiment in a picture where the particle remains as such from the moment of emission until the moment of absorption, but is 'informed' about the probability of different paths by the pilot wave (i.e. it is guided by the pilot wave) [5]. The path of the particle within the period between emission and absorption is unknown (if no additional measurement is performed that would spoil the experiment), that is why the particle itself is termed 'hidden' during the experiment. The theory is 'non-local, realistic' along the classification of Bell [6]. Non-local means that physical observables are represented by gradient operators acting on fields in space and time. Realistic means that physical observables have well-defined values (not necessarily 'exact', but subject to Heisenberg's uncertainty) independent of a measurement. This contrasts to 'local, realistic' classical theories; Newton's mechanics and general relativity; and the 'non-local, probabilistic' Copenhagen interpretation of quantum mechanics.

de Broglie, the father of the wave interpretation of elementary particles, presented his ideas - how to construct stable elementary particles and their interaction as wave phenomena - first at the Solvay conference 1927. For a recent review and details, see [7]. The solution of a linear Schrödinger-type wave equation couples to the solution of a non-linear wave equation that has localised amplitudes interpreted as massive particles, the $u$ wave. Both waves are connected by their phases, which is why the pilot wave can guide the particle. According to Bohm's work, the Schrödinger equation for the $\psi$ wave can be decomposed into two equations, for the amplitude 
( $R$ in Bohm's notation) and for the phase ( $S$ in Bohm's notation). The probability of finding a particle $\left(P=R^{2}\right)$ relates to the probability in the Copenhagen interpretation. Using the phase $S$, the velocity of the particle can be found as $v_{\mathrm{p}}=-c^{2} \nabla S / \partial_{t} S$. This can be seen in analogy to the particles in water waves, which make loops that gradually advance in the direction of wave propagation [8].

Why treat matter as coupled waves? Why not simply accept the existence of elementary particles as point masses? The most prominent opponent of the dualism of mass and space was Einstein, who worked for long on a 'unitary' or 'monistic' view of the physical world, as reported in [7]:

'[...] Einstein [...] had a very similar objective in the framework of his theory of general relativity in the 20s: the postulate of geodesics would not be an extra hypothesis, but would be obeyed, de facto, by peaked solutions of Einstein's non-linear equations moving on a weakly varying metric background. This idea presents many deep similarities with de Broglie's guidance equation, which lies at the heart of the de Broglie-Bohm hidden variable theory [1, 2]. In fact, Bohm-de Broglie trajectories are the counterpart of geodesic trajectories in Einstein's unitarian version of general relativity ....'

Today, it is almost fully accepted that elementary particles cannot be considered as point masses and a nonlocal theory, such as the dBB double-solution program, is needed. An appropriate quantum field theory that is based on elementary space quanta with finite dimensions, e.g. strings [9] or branes [10], had not been worked out at that time and is still missing in a concise form today. The double-solution program offers an elegant solution to this problem because it combines a linear Schrödingertype wave equation for a $\psi$ wave with a non-linear wave equation for a $u$ wave describing the particle.

The main difficulty, the reason why the theory did not prevail, was the construction of the $u$ wave representing the particle, or other quantum-mechanical objects. Although de Broglie's primary idea had been the description of matter as a wave phenomenon, the double-solution program is based on the traditional understanding of 'particles' and 'space' as separate elements of matter. Recently, the second author developed a monistic view of matter, called 'quantum-phase-field (PF) concept' [11] (The term 'phase-filed' denotes a special 'soliton' solution in material physics and pattern formation, see [12-14]), treating space-time and mass as two manifestations of matter with dualistic character. In the quantum-PF concept, a special field solution, which we will call 'doublon' in the following, forms the elementary building block of matter.
It unifies the aspects of particles and space. Only after publication, his attention was called to the similarity with the $\mathrm{dBB}$ double-solution program, which shall be elaborated in the present article.

Here, we have to link to another development, which began in the 1960s in the analysis of non-linear wave equations that are related to a completely different phenomenon: shallow water waves in channels and ocean shores. The problem has been described by Korteweg and de Vries (KdV) in 1896 [15]. Solutions of this type of nonlinear wave equations are characterised by a self-similar shape of wave packets, nowadays known as 'solitons' (for review, see [16, 17]). The soliton solutions had been unknown to de Broglie and Bohm in the beginning of the 1960s.

Today, the 'soliton' appears in a new application, the PF theory [12-14], which is based on a soliton solution of a special non-linear wave equation derived from a Ginzburg-Landau functional. The PF theory is applied to investigate pattern formation in condensed matter physics and materials science. The pattern here is the morphology of a 'phase' in real space and its evolution, therefrom the name. A phase is identified by an order parameter $\phi$, called PF variable. It is a state of matter with a discontinuous property relation to the other phases in the sense of Landau [18]. We shall not confuse this interpretation of a 'phase' with the phase of a wave, although we will show that, indeed, it can have a similar formal meaning if we speak about localised wave solutions, i.e. solitons.

One distinguishes primarily two sets of 'solitons', as depicted in Figure 1, with the eigen-coordinate $\xi$, space coordinate $x$, time coordinate $t$, size $\eta$, and velocity $v$.

Figure 1a depicts the classical soliton as an excitation against a homogeneous background. Besides numerous applications in wave mechanics (see [16] for an exhaustive review at its time), it has been proposed as a template to describe elementary particles [17]. The original soliton is a 1-dimensional or planar 2-dimensional solution of a spatial non-linear wave equation. True 3-dimensional realisations have been shown to be mathematically impossible
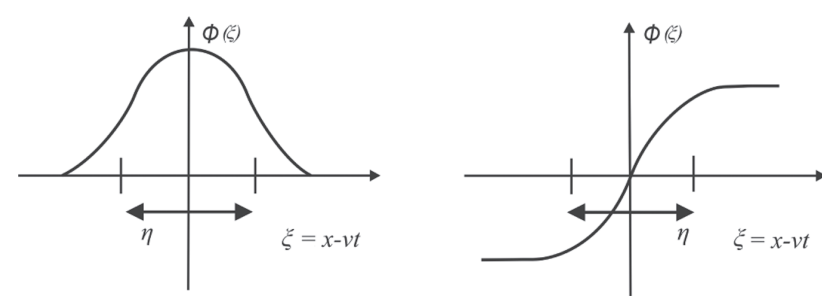

Figure 1: Soliton in 1+1 dimension. (a) Symmetric soliton. (b) Half-sided soliton as an integral form of the symmetric soliton. $\eta$ is the characteristic size of the wave. 
[19]. The construction of 'particles' then is only possible in a spherical symmetric approximation (see, e.g. [20]), which, by construction, prevents investigating scattering events. It has also been realised early on that the intrinsic length scale of the soliton, its size $\eta$, leads to a "natural mode of quantisation' [21]. The latter statement leads back to the dBB program. This connection has been little addressed in the literature, but is clearly pointed out by Colin and Durt [7].

Figure $1 \mathrm{~b}$ shows a solution of an integral form of the classical KdV-type equation, which introduces parity in the space coordinate. We will later, in Section 6, combine two of these 'half-sided solitons', a 'right-moving soliton' and a 'left-moving soliton', to an antisymmetric pair, which we call 'doublon' (The expression 'doublon' has been coined originally by Ihle and Mueller-Krumbhaar [22] in the context of a pair morphology in pattern formation). The doublon will serve (i) to define massive particles with positive energy by gradient operators and (ii) define 'space' as the distance between the particles, attributed by negative energy.

We will proceed as follows:

- Describe the relation of the PF equation to the $\mathrm{dBB}$ double-solution program.

- Present Lax pairs that couple two wave functions similar to the dBB program.

- Construct 'doublons' from an antisymmetric pair of half-sided left-/right-moving solitons from the PF equation.

- Construct a 'doublon network' in $1+1+2$ dimensions that embeds massive particles.

- Propose a generalisation of the concept to charged particles and fields.

- Discuss limitations and perspectives of the current approach.

\section{PF as a Toy Problem for the dBB Double-Solution Program}

\subsection{PF Equation for the Phase of a Wave Function}

The PF order parameter $\phi$ can be considered in analogy to the phase $S$ in the dBB double-solution program [4]. de Broglie and Bohm derived equations for the phase and for the amplitude, taking the pilot wave in the form $\psi=R e^{i S}$ and substituting it into the Schrödinger equation. Here, we solve the inverse problem and seek for an equation for a $\psi$ wave, whose phase evolves according to a PF equation.
In order to be consistent, we define $\phi$ as the phase of a wave function $\psi$ with an amplitude $a_{\psi}$ as

$$
\psi=a_{\psi} e^{i \phi} .
$$

Then, we consider the linear PF equation as an evolution equation for the phase:

$$
\tau \partial_{t} \phi=\eta^{2} \partial_{x x} \phi+\phi+\eta e \partial_{x} \phi,
$$

where $\partial_{t}=\frac{\partial}{\partial t}, \partial_{x}=\frac{\partial}{\partial x}$ and $\partial_{x x}=\frac{\partial^{2}}{\partial x^{2}}$.

Here, $\tau$ is a kinetic parameter with dimension of time; $\eta$ is a characteristic length to be identified with the size of the resulting soliton solution (see Fig. 1), which is also called 'interface width' in the PF model; and $e$ is a dimensionless parameter related to the velocity of transport. The simple solution of this equation is $\phi=e^{i \theta}$ with $\theta=$ $\frac{x+v_{\phi} t}{\eta}$ and the velocity $v_{\phi}=\frac{\eta e}{\tau}$. For this kind of solution, the PF equation can be separated into two equations: the advection equation $\partial_{t} \phi=v_{\phi} \partial_{x} \phi$ and the equation for the self-similar shape of the soliton.

From the equation for the phase, we can reconstruct the equation for $\psi$. The following relations can be useful as parts of the sought equation:

$$
\begin{aligned}
\partial_{t} \psi= & \partial_{t}\left(a_{\psi} e^{i \phi}\right)=\frac{\partial_{t} a_{\psi}}{a_{\psi}} \psi+i\left(\partial_{t} \phi\right) \psi \\
= & \frac{\partial_{t}\left(a_{\psi}^{2}\right)}{2 a_{\psi}^{2}} \psi+i\left(\partial_{t} \phi\right) \psi, \\
\partial_{x x} \psi= & 2 i \frac{\partial_{x} a_{\psi}}{a_{\psi}}\left(\partial_{x} \phi\right) \psi+i^{2}\left(\partial_{x} \phi\right)^{2} \psi \\
& +i\left(\partial_{x x} \phi\right) \psi+\frac{\partial_{x x} a_{\psi}}{a_{\psi}} \psi \\
= & {\left[-\left(\partial_{x} \phi\right)^{2}+\frac{\partial_{x x} a_{\psi}}{a_{\psi}}\right] \psi } \\
& +i\left[2 \frac{\partial_{x} a_{\psi}}{a_{\psi}}\left(\partial_{x} \phi\right)+\partial_{x x} \phi\right] \psi .
\end{aligned}
$$

With these relations, the following form of the evolution equation for $\psi$ can be suggested:

$$
\tau \partial_{t} \psi=\eta^{2} \partial_{x x} \psi+i \phi \psi+i \eta e_{0}\left(\partial_{x} \phi\right) \psi
$$

where $e_{0}$ is a parameter to be defined. By the substitution of the solution (1) in this equation and collecting the necessary imaginary terms, we obtain the equation for the phase (2) with $e=e_{0}$. 
The equation for the amplitude is the collection of the remaining terms, i.e.

$$
\begin{aligned}
\tau \frac{\partial_{t}\left(a_{\psi}^{2}\right)}{2 a_{\psi}^{2}}= & -\eta^{2}\left(\partial_{x} \phi\right)^{2}+\eta^{2} \frac{\partial_{x x} a_{\psi}}{a_{\psi}} \\
& +2 i \eta^{2} \frac{\partial_{x} a_{\psi}}{a_{\psi}}\left(\partial_{x} \phi\right)
\end{aligned}
$$

After substitution $\frac{\partial_{x x} a_{\psi}}{a_{\psi}}=\frac{\partial_{x x}\left(a_{\psi}^{2}\right)}{2 a_{\psi}^{2}}-\frac{\left(\partial_{x} a_{\psi}\right)^{2}}{a_{\psi}^{2}}$, we obtain

$$
\begin{aligned}
\tau \partial_{t}\left(a_{\psi}^{2}\right)= & \eta^{2} \partial_{x x}\left(a_{\psi}^{2}\right)-2 \eta^{2}\left(\partial_{x} \phi\right)^{2} a_{\psi}^{2}-2 \eta^{2}\left(\partial_{x} a_{\psi}\right)^{2} \\
& +4 i \eta^{2} a_{\psi}\left(\partial_{x} a_{\psi}\right)\left(\partial_{x} \phi\right)
\end{aligned}
$$

As this equation can be considered a PF-like equation, it can be divided into two equations:

$$
\begin{gathered}
\tau \partial_{t}\left(a_{\psi}^{2}\right)=2 i \eta^{2}\left(\partial_{x} \phi\right) \partial_{x}\left(a_{\psi}^{2}\right) \\
\eta^{2} \partial_{x x}\left(a_{\psi}^{2}\right)=2 \eta^{2}\left(\partial_{x} \phi\right)^{2} a_{\psi}^{2}+2 \eta^{2}\left(\frac{\partial_{x} a_{\psi}}{a_{\psi}}\right)^{2} a_{\psi}^{2} .
\end{gathered}
$$

Equation (7) is the advection equation

$$
\partial_{t}\left(a_{\psi}^{2}\right)=v_{a_{\psi}} \partial_{x}\left(a_{\psi}^{2}\right)
$$

with the velocity

$$
v_{a_{\psi}}=\frac{2 i \eta^{2}}{\tau} \partial_{x} \phi
$$

Equation (8) defines the self-similar shape and can be written as

$$
\eta_{a_{\psi}}^{2} \partial_{x x}\left(a_{\psi}^{2}\right)=-a_{\psi}^{2}
$$

with

$$
\eta_{a_{\psi}}^{2}=-\frac{1}{2}\left(\left(\partial_{x} \phi\right)^{2}+\left(\frac{\partial_{x} a_{\psi}}{a_{\psi}}\right)^{2}\right)^{-1}
$$

A solution of this equation is $a_{\psi}^{2}(x, 0)=e^{i x / \eta_{a_{\psi}}}$ or $a_{\psi}(x, 0)=e^{i x /\left(2 \eta_{a_{\psi}}\right)}$.

Using the advection equation (7), we obtain a total solution for the amplitude $a_{\psi}^{2}(x, t)=e^{i \theta_{a}}$ with $\theta_{a}=$ $\frac{x+v_{a_{\psi}} t}{\eta_{a_{\psi}}}$.

Assuming that the velocity of the amplitude $v_{a_{\psi}}=$ $\frac{2 i \eta^{2}}{\tau} \partial_{x} \phi$ is equal to the particle velocity $v_{\mathrm{p}}=-c^{2} \frac{\partial_{x} \phi}{\partial_{t} \phi}$, and substituting $\partial_{t} \phi=\frac{i e \phi}{\tau}$, we can define the characteristic length $\eta$ from $\eta^{2}=\frac{c^{2} \tau^{2}}{2 e \phi}$. For the mean value $\bar{\phi}=\frac{1}{2}, \eta \approx$ $\frac{c \tau}{\sqrt{e}}$. The particle velocity is then defined as $v_{a_{\psi}}=\frac{2 \phi c}{\sqrt{e}} \approx$ $\frac{c}{\sqrt{e}}=v_{\mathrm{p}}$, and the velocity of the phase $\phi$ is defined as $v_{\phi}=c \sqrt{e}$. Moreover, by substitution in (12) $\frac{\partial_{x} a_{\psi}}{a_{\psi}}=\frac{i}{2 \eta_{a_{\psi}}}$ and $\partial_{x} \phi=\frac{i \bar{\phi}}{\eta}$, we get $\eta_{a_{\psi}}=\eta$.

According to our results, the particle velocity decreases with increasing $e$, whereas the phase velocity increases. Both velocities approach $c$ when $e$ decreases. Moreover, $v_{\mathrm{a}_{\psi}} v_{\phi}=c^{2}$ in accordance with the dBB theory.

In quantum mechanics, the momentum (i.e. the particle velocity) should be inversely proportional to the wavelength, identified with the characteristic length $p \sim 1 / \eta$, and directly proportional to the particle velocity. Hence, if we assume $\tau=\tau_{0} e, \eta$ will be proportional to $\sqrt{e}$ and $p$ as well as $v_{\mathrm{a}_{\psi}}$ will be proportional to $1 / \sqrt{e}$ according to expectations. Based on that result, the characteristic length can be defined as $\eta=c \tau_{0} \sqrt{e}$.

In summary, the particle is described by the amplitude function $a_{\psi}(x, t)$ as a 1-dimensional singularity of size $\eta$ moving with the velocity $v_{a_{\psi}}$, which is the solution of the PF-like equation (6). This amplitude corresponds to the $u$ wave in the $\mathrm{dBB}$ theory and $\phi$ is the pilot wave for the amplitude. Meanwhile, the dynamics of $\phi$ is governed by (2). Finally, we show that (2) can be transformed to the Schrödinger equation with a constant potential. Dividing the two first terms on the right-hand side of (2) by $2 i$ (this is allowed because the PF equation can be separated into two different equations), we get

$$
\tau \partial_{t} \phi=\frac{\eta^{2}}{2 i} \partial_{x x} \phi+\frac{\phi}{2 i}+\eta e \partial_{x} \phi .
$$

Then, by substitution $\tau_{0}=\frac{\hbar}{m c^{2}}$ and $\partial_{x} \phi=\frac{i \phi}{\eta}$, we obtain the Schrödinger-type equation:

$$
\partial_{t} \phi=\frac{\hbar}{2 i m} \partial_{x x} \phi+\frac{i U_{0}}{\hbar} \phi,
$$

with $U_{0}=m c^{2}\left(1-\frac{1}{2 e}\right)$. Here, $m$ is the mass of the particle and $\hbar$ is Plank's constant.

\subsection{PF Equation for the Pilot Wave}

In this section, we consider the transformed PF equation (14) as a Schrödinger-type equation with a given potential. The PF order parameter $\phi$ will be now considered as the pilot $\psi$ wave. We rewrite (13) as

$$
\tau \partial_{t} \psi=\frac{\eta^{2}}{2 i} \partial_{x x} \psi+\frac{\psi}{2 i}+\eta e \partial_{x} \psi
$$

and define $\psi=a_{\psi} e^{i S}$ with $S=\frac{x+v_{\psi} t}{\eta}$ and $v_{\psi}=\frac{\partial_{t} S}{\partial_{x} S}=\frac{e \eta}{\tau}$. Then, we assume that the pilot wave $\psi$ is the phase of a superwave

$$
\Phi=a_{\Phi} e^{i \psi}
$$


From (15), we can reconstruct the equation for $\Phi$. Using the terms (3) (where we replace $\psi$ by $\Phi, a_{\psi}$ by $a_{\Phi}$, and $\phi$ by $\psi$ ), i.e.

$$
\begin{aligned}
\partial_{t} \Phi= & \frac{\partial_{t} a_{\Phi}}{a_{\Phi}} \Phi+i\left(\partial_{t} \psi\right) \Phi \\
\partial_{x x} \Phi= & {\left[-\left(\partial_{x} \psi\right)^{2}+\frac{\partial_{x x} a_{\Phi}}{a_{\Phi}}\right] \Phi } \\
& +i\left[2 \frac{\partial_{x} a_{\Phi}}{a_{\Phi}}\left(\partial_{x} \psi\right)+\partial_{x x} \psi\right] \Phi,
\end{aligned}
$$

the following form of the evolution equation for $\Phi$ can be suggested:

$$
\begin{aligned}
\tau \partial_{t} \Phi= & \frac{\eta^{2} \partial_{x x} \Phi}{2 i}+\frac{\psi \Phi}{2}+\frac{f\left(a_{\Phi}\right) \Phi}{2 i} \\
& +i \eta e_{0}\left(\partial_{x} \psi\right) \Phi+\eta e_{0}^{a} \frac{\partial_{x} a_{\Phi}}{a_{\Phi}} \Phi
\end{aligned}
$$

where $e_{0}$ and $e_{0}^{a}$ are parameters responsible for the motion of $\psi$ and $a_{\Phi}$, respectively, which have to be defined. The term $\frac{f\left(a_{\Phi}\right) \Phi}{i}$ is added by analogy to $\psi \Phi$. By substituting the $\Phi$ wave (16) in this equation and collecting the imaginary terms in (17), the equation for $\psi$ (15) can be reconstructed with

$$
e=e_{0}-i \eta \frac{\partial_{x} a_{\Phi}}{a_{\Phi}} .
$$

The equation for the amplitude is the collection of the real terms in (17), i.e.

$$
\begin{aligned}
\tau \frac{\partial_{t} a_{\Phi}}{a_{\Phi}}= & \frac{\eta^{2}}{2 i} \frac{\partial_{x x} a_{\Phi}}{a_{\Phi}}+\frac{f\left(a_{\Phi}\right)}{2 i} \\
& -\frac{\eta^{2}}{2 i}\left(\partial_{x} \psi\right)^{2}+\eta e_{0}^{a} \frac{\partial_{x} a_{\Phi}}{a_{\Phi}} .
\end{aligned}
$$

This equation can be treated as a set of two equations:

$$
\begin{gathered}
\tau \partial_{t} a_{\Phi}=\eta e_{0}^{a} \partial_{x} a_{\Phi}+\frac{i \eta^{2}}{2}\left(\partial_{x} \psi\right)^{2}\left(\frac{a_{\Phi}}{\partial_{x} a_{\Phi}}\right) \partial_{x} a_{\Phi}, \\
\eta_{a_{\Phi}}^{2} \partial_{x x} a_{\Phi}=-a_{\Phi} f\left(a_{\Phi}\right),
\end{gathered}
$$

where $\eta_{a_{\Phi}}=\eta$. By assumption $f\left(a_{\Phi}\right)=1$, a solution of (22) reads $a_{\Phi}(x, 0)=e^{i x / \eta a_{\Phi}}$. Substituting $\frac{\partial_{x} a_{\Phi}}{a_{\Phi}}=\frac{i}{\eta}$, we can rewrite the advection equation (21) in the form

$$
\partial_{t} a_{\Phi}=v_{a_{\Phi}} \partial_{x} a_{\Phi},
$$

with $v_{a_{\Phi}}=-\frac{\eta e^{a}}{\tau}$ and

$$
e^{a}=e_{0}^{a}+\frac{\eta^{2}}{2}\left(\partial_{x} \psi\right)^{2} .
$$

The full solution for the amplitude is $a_{\Phi}(x, t)=e^{i \theta_{a}}$ with $\theta_{a}=\frac{x-v_{a_{\Phi}} t}{\eta_{a_{\Phi}}}$. The amplitude can be interpreted as a particle moving with the velocity $v_{a_{\Phi}}=v_{\mathrm{p}}=-c^{2} \frac{\partial_{\partial} S}{\partial_{t} S}$. From this, we can define the characteristic length as $\eta^{2}=$ $\frac{c^{2} \tau^{2}}{e e^{a}}$. The particle velocity is then defined as $v_{a_{\Phi}}=c \sqrt{\frac{e^{a}}{e}}$, and the velocity of the wave $\psi$ is defined as $v_{\psi}=c \sqrt{\frac{e}{e^{a}}}$. Here, we have restriction for the particle velocity $e^{a} \leq e$. By substituting $\frac{\partial_{x} a_{\Phi}}{a_{\Phi}}=\frac{i}{\eta_{a_{\Phi}}}$ in (19), we obtain $e=e_{0}+$ $\eta / \eta_{a_{\Phi}}=e_{0}+1$.

Note that the phase and the amplitude of the wave $\psi=$ $a_{\psi} e^{i S}$ evolve according to $\mathrm{dBB}$ equations [4], which are the Hamilton-Jacobi equation for the phase and the continuous equation for the amplitude. The difference between the dBB program and our solution is that in the present variant, the amplitude of the superwave $a_{\Phi}$ is representing the particle that was previously considered as the $u$ wave. The guidance occurs as before by the dBB quantum force, which acts on the phase $S$, which then changes the particle velocity. Hence, the function $\psi$ is the pilot wave for $a_{\Phi}$.

Finally, we can rewrite (15) in physical units. By substitution $\tau=\tau_{0} e, \tau_{0}=\frac{\hbar}{m c^{2}}$, and $\eta=\tau_{0} v_{\psi}$, we obtain

$$
\partial_{t} \psi=\frac{\hbar v_{\psi}^{2}}{m c^{2}} \partial_{x x} \psi+\frac{m c^{2}}{\hbar} \psi+v_{\psi} \partial_{x} \psi
$$

Then, multiplying the two first terms on the right-hand side by $\frac{c^{2}}{\left(2 i v_{\psi}^{2}\right)}$ and using $\partial_{x} \psi=i \frac{\psi}{\eta}$, we get the Schrödingertype equation:

$$
\partial_{t} \psi=\frac{\hbar}{2 i m} \partial_{x x} \psi+i \frac{U_{0}}{\hbar} \psi,
$$

with $U_{0}=m c^{2}\left(1-\frac{c^{2}}{2 v_{\psi}^{2}}\right)$. In the limit $v_{\psi} \rightarrow \infty, U_{0} \rightarrow$ $m c^{2}$.

\section{Lax Method for the Solution of Wave Equations}

The solution of a scattering problem in quantum mechanics is associated with the linear Lax operators $\mathcal{L}$ and $\mathcal{A}$, called Lax pair, which satisfy the Lax equation [23]:

$$
\mathcal{L}_{t}+[\mathcal{L}, \mathcal{A}]=0
$$

This equation should reproduce a partial differential equation (PDE) for a function $\phi$, whereas the Lax operators are applied to a function $\psi$, which is the solution of the Schrödinger-type equations. Therefore, the function $\psi$ is coupled to the function $\phi$ by the Lax pair. Hence, the Lax 
method can describe the coupling of two wave functions in the dBB double-solution theory [4].

In order to find the solution of the PDE, one should solve the forward and inverse scattering problems. The forward problem is solved by the first and second Lax equations with the eigenvalue $\lambda$ :

$$
\begin{gathered}
\mathcal{L} \psi=\lambda \psi, \\
\partial_{t} \psi=\mathcal{A} \psi .
\end{gathered}
$$

The inverse scattering problem is solved by calculating the Gelfand-Levitan-Marchenko integral (31). The function .. is constructed from the formula

$$
\phi(x, t)=-2 \frac{\partial}{\partial x} K(x, x, t),
$$

where $K(x, y, t)$ is the solution of the linear integral equation

$$
K(x, y)+F(x+y)+\int_{x}^{+\infty} K(x, z) F(y+z) \mathrm{d} z=0,
$$

defined for a fixed time $t$ and $F$ is the function that is based on the solution of the Lax equations for $\psi$.

\subsection{Lax Pair for the Phase and Amplitude Equations}

Now we show that the phase equation (2) can be reconstructed by the Lax method applied to a function $\psi$ whose evolution equation is described by the second Lax equation.

We choose the Lax operators as

$$
\begin{aligned}
& \mathcal{L}=\tau \eta \partial_{x}+\tau \phi+\tau \eta i \partial_{x}+\tau i \phi, \\
& \mathcal{A}=\frac{\eta^{2}}{\tau} \partial_{x x}+i \frac{\phi}{\tau}+i \frac{\eta e_{0}}{\tau} \partial_{x} \phi .
\end{aligned}
$$

The non-zero components of the Lax equation (27) have the terms

$$
\begin{aligned}
{\left[\phi, \partial_{x x}\right] \psi } & =\phi \psi_{x x}-\partial_{x x}(\phi \psi) \\
& =\phi \psi_{x x}-\partial_{x}\left(\phi_{x} \psi+\phi \psi_{x}\right) \\
& =\phi \psi_{x x}-\left(\phi_{x x} \psi+\phi_{x} \psi_{x}+\phi_{x} \psi_{x}+\phi \psi_{x x}\right) \\
& =-\phi_{x x} \psi-2 \phi_{x} \psi_{x} \\
{\left[\partial_{x}, \phi\right] \psi } & =\partial_{x}(\phi \psi)-\phi \psi_{x}=\phi_{x} \psi, \\
{\left[\partial_{x}, \phi_{x}\right] \psi } & =\phi_{x x} \psi .
\end{aligned}
$$

Using $\phi=\phi_{0} e^{i \theta}$ and $\psi=\psi_{0} e^{i \theta}$, we can substitute $\phi_{x}=(\log \phi)_{x} \phi$ and $\psi_{x}=(\log \psi)_{x} \psi$ and get $\phi_{x x} \psi=$ $i \theta_{x} \phi_{x} \psi, \phi_{x} \psi=i \theta_{x} \phi \psi$, and $\phi_{x} \psi_{x}=i \theta_{x} \phi_{x} \psi$. Then, we assume $\theta_{x}=1 / \eta$. The components of the Lax equation including real and imaginary terms become

$$
\begin{aligned}
\eta^{2}\left[\phi, \partial_{x x}\right] \psi & =-\eta^{2} \phi_{x x} \psi-2 \eta^{2} \phi_{x} \psi_{x} \\
& =-\eta^{2} \phi_{x x} \psi-2 i \eta \phi_{x} \psi, \\
\eta^{2}\left[i \phi, \partial_{x x}\right] \psi & =-i \eta^{2} \phi_{x x} \psi-2 i \eta^{2} \phi_{x} \psi_{x} \\
& =-i \eta^{2} \phi_{x x} \psi+2 \eta \phi_{x} \psi, \\
\eta\left[\partial_{x}, i \phi\right] \psi=i \eta \phi_{x} \psi & =-\phi \psi, \\
\eta\left[i \partial_{x}, i \phi\right] \psi=-\eta \phi_{x} \psi & =-i \phi \psi, \\
\eta^{2} e_{0}\left[\partial_{x}, i \phi_{x}\right] \psi & =i \eta^{2} e_{0} \phi_{x x} \psi=-\eta e_{0} \phi_{x} \psi, \\
\eta^{2} e_{0}\left[i \partial_{x}, i \phi_{x}\right] \psi & =-\eta^{2} e_{0} \phi_{x x} \psi=-i \eta e_{0} \phi_{x} \psi .
\end{aligned}
$$

After substitution in (27), we obtain

$$
\begin{aligned}
\tau \partial_{t} \phi & =\eta^{2} \partial_{x x} \phi+\phi+\left(e_{0}-2\right) \eta \partial_{x} \phi, \\
i \tau \partial_{t} \phi & =i \eta^{2} \partial_{x x} \phi+i \phi+i\left(e_{0}+2\right) \eta \partial_{x} \phi .
\end{aligned}
$$

These equations recover the $\mathrm{PF}$ equation (2) for $e=$ $e_{0} \pm 2$.

The first Lax equation for the $\psi$ function with the eigenvalues $\lambda_{1}$ and $\lambda_{2}$ is

$$
i \eta \partial_{x} \psi+\phi \psi+\eta \partial_{x} \psi+i \phi \psi=\lambda_{1} \psi+i \lambda_{2} \psi,
$$

and the second Lax equation is

$$
\tau \partial_{t} \psi=\eta^{2} \partial_{x x} \psi+i \phi \psi+i \eta e_{0}\left(\partial_{x} \phi\right) \psi
$$

which recovers (4) for the $\psi$ wave one to one.

The Lax pair can also be found for the waves in Section 2.1 in the form $\phi=e^{i \theta}$ and $\psi=a_{\psi} e^{i \phi}$, where $\phi$ is the phase of the pilot wave $\psi$. Doing so, we deviate from the Lax method, which requires that the phase of both functions should be equal and time independent. However, we use again the assumption that $\theta_{x}=1 / \eta$. We show now that the following Lax pair can reproduce (2) and (6):

$$
\begin{aligned}
\mathcal{L}= & \tau \eta \partial_{x}+\tau \phi+\tau \frac{2 a_{\psi}(x, t)}{a_{\psi}}+\tau \eta i \partial_{x} \\
& +\tau i \phi+\tau i \frac{2 a_{\psi}(x, t)}{a_{\psi}} \\
\mathcal{A}= & \frac{\eta^{2}}{\tau} \partial_{x x}+i \frac{\phi}{\tau}+i \frac{\eta e_{0}}{\tau} \partial_{x} \phi .
\end{aligned}
$$


The non-zero components of the Lax equation have the terms

$$
\begin{aligned}
\eta^{2}\left[\phi, \partial_{x x}\right] \psi= & -\eta^{2} \phi_{x x} \psi-2 \eta^{2} \phi_{x} \psi_{x} \\
= & -\eta^{2} \phi_{x x} \psi-2 i \eta^{2}\left(\phi_{x}\right)^{2} \psi \\
& -2 \eta^{2} \frac{\partial_{x} a_{\psi}}{a_{\psi}} \phi_{x} \psi, \\
\eta\left[\partial_{x}, i \phi\right] \psi= & i \eta \phi_{x} \psi=-\phi \psi, \\
\eta^{2} e_{0}\left[\partial_{x}, i \phi_{x}\right] \psi= & i \eta^{2} e_{0} \phi_{x x} \psi=-\eta e_{0} \phi_{x} \psi, \\
\frac{\eta^{2}}{a_{\psi}}\left[i a_{\psi}(x), \partial_{x x}\right] \psi= & -i \eta^{2} \frac{\partial_{x x} a_{\psi}}{a_{\psi}} \psi-2 i \eta^{2} \frac{\partial_{x} a_{\psi}}{a_{\psi}} \psi_{x} \\
= & -i \eta^{2} \frac{\partial_{x x} a_{\psi}}{a_{\psi}} \psi-2 i \eta^{2} \frac{\left(\partial_{x} a_{\psi}\right)^{2}}{a_{\psi}^{2}} \psi \\
& +2 \eta^{2} \frac{\partial_{x} a_{\psi}}{a_{\psi}} \phi_{x} \psi .
\end{aligned}
$$

Here, we used $\psi_{x}=\frac{\partial_{x} a_{\psi}}{a_{\psi}} \psi+i \phi_{x} \psi$ and normalised the $a_{\psi}$ operator by $a_{\psi}$. We also do not write the similar complex conjugate terms to be compact.

Substituting all non-zero components in (27) and collecting imaginary and real terms, we obtain two equations:

$$
\begin{aligned}
\tau \partial_{t} \phi= & \eta^{2} \partial_{x x} \phi+\phi+e_{0} \eta\left(\partial_{x} \phi\right), \\
\tau \partial_{t} a_{\psi}^{2}= & \eta^{2} \partial_{x x} a_{\psi}^{2}-2 \eta^{2}\left(\partial_{x} \phi\right)^{2} a_{\psi}^{2}-2 \eta^{2}\left(\partial_{x} a_{\psi}\right)^{2} \\
& +4 i \eta^{2} a_{\psi}^{2} \frac{\partial_{x} a_{\psi}}{a_{\psi}}\left(\partial_{x} \phi\right),
\end{aligned}
$$

which for $e=e_{0}$ lead to (2) and (6) for the phase and the amplitude, whereas the evolution equation for the wave $\psi$ is in the same form as before [see (37)], i.e. recovers (4) for the $\psi$ wave. Hence, we have shown that the Lax method can be used also for the coupling of three functions, one of which is the function of two others. Note that in this case, $\phi$ is the pilot wave for $R$.

The Lax pair for (15)-(20) can be found in the same manner by adding the necessary terms in the operators $\mathcal{L}$ and $\mathcal{A}$. Thus, for the ansatz $\psi=e^{i S}, \Phi=a_{\Phi} e^{i \psi}$, and $\partial_{x} S=1 / \eta$, the Lax pair for (15)-(20) have the form

$$
\begin{aligned}
\mathcal{L}= & \tau \eta \partial_{x}+\tau \psi+\tau \frac{2 a_{\Phi}(x, t)}{a_{\Phi}}+\tau \eta i \partial_{x} \\
& +\tau i \psi+\tau i \frac{2 a_{\Phi}(x, t)}{a_{\Phi}}, \\
\mathcal{A}= & \frac{\eta^{2}}{2 i \tau} \partial_{x x}+\frac{i \psi}{2 i \tau}+i \frac{\eta e_{0}}{\tau} \partial_{x} \psi \\
& +\frac{f\left(a_{\Phi}\right)}{2 i \tau}+\frac{\eta e_{0}^{a}}{\tau} \frac{\partial_{x} a_{\Phi}}{a_{\Phi}} .
\end{aligned}
$$

Finally, the Lax equation (27) recover (15) and (20) for $\psi$ and $a_{\Phi}$, and first and second Lax equations [(28) and
(29)] recover (18) for $\Phi$. Note that, in this case, the function $\psi$ is the pilot wave for $a_{\Phi}$.

\subsection{Lax Pairs for the Non-Linear PF Equation}

We consider a non-linear wave equation for a wave $\phi$, also known in material physics as the 'PF equation' with double obstacle (DO) potential [13]:

$$
\tau \partial_{t} \phi=\eta^{2} \partial_{x x} \phi-f_{\phi}(\phi)+\eta e \partial_{x} \phi,
$$

where $f_{\phi}(\phi)$ is a non-linear scalar function in $\phi$, which is the first derivative of the DO potential:

$$
f(\phi)=\frac{1}{2}|\phi(1-\phi)| .
$$

The non-linearity is hidden in the break points $\phi=0$ and $\phi=1$. We will argue later that this ansatz is responsible for the localisation of the wave solution. One can use here, without loss of generality, the functional form:

$$
f_{\phi}(\phi)=\left(\frac{1}{2}-\phi\right) \operatorname{signum}(\phi(1-\phi))
$$

which means $f_{\phi}(\phi)=\left(\frac{1}{2}-\phi\right)$ for $0 \leq \phi \leq 1$ and $f_{\phi}(\phi)=$ $\left(\phi-\frac{1}{2}\right)$ otherwise.

A solution of (42) with the potential (43) is, e.g.

$$
\phi(x, t)=\frac{1}{2} \sin \left(\frac{x-v t}{\eta}\right)+\frac{1}{2},
$$

for $-\frac{\eta}{2}-v t<x<\frac{\eta}{2}-v t$ for a half-wave traveling with velocity $v=\frac{\eta e}{\tau}$ (see also [11]). We see that the non-linear absolute operator, |.|, cuts out one localised half wave from the periodic sinus wave. Although the solution is known, we will investigate the possibility to find a solution of this PDE by the 'inverse scattering method' and demonstrate connections between two wave functions $\phi$ and $\psi$. For this aim, we will find the Lax pairs and show that (42) can be reproduced by the Lax representation.

\subsubsection{Variant I}

We choose the Lax operators in the form

$$
\begin{aligned}
& \mathcal{L}=\tau \eta \partial_{x}+\tau \phi, \\
& \mathcal{A}=\frac{\eta^{2}}{\tau} \partial_{x x}+\frac{V(x)}{\tau \eta}+\frac{e_{0}}{\tau} \phi,
\end{aligned}
$$

where $V(x)=\int^{x} f_{\phi}(\phi) \mathrm{d} x$ and $e_{0}$ is a constant to be defined. This type of equation was suggested first by Zakharov and Shabat [24]. 
The non-zero components of the Lax equation are

$$
\begin{aligned}
{\left[\partial_{x}, V(x)\right] \psi } & =\partial_{x}(V(x) \psi)-V(x) \psi_{x} \\
& =\psi \partial_{x} V(x)+V(x) \psi_{x}-V(x) \psi_{x} \\
& =f_{\phi}(\phi) \psi ;
\end{aligned}
$$$$
\left[\phi, \partial_{x x}\right] \psi=-\phi_{x x} \psi-2 \phi_{x} \psi_{x}
$$$$
\left[\partial_{x}, \phi\right] \psi=\partial_{x}(\phi \psi)-\phi \psi_{x}=\phi_{x} \psi \text {. }
$$

After substitution in (27), we obtain

$$
\begin{aligned}
& \tau \partial_{t} \phi \psi+f_{\phi}(\phi) \psi-\eta^{2} \partial_{x x} \phi \psi \\
& \quad-2 \eta^{2} \partial_{x} \phi \partial_{x} \psi+e_{0} \eta \partial_{x} \phi \psi=0 .
\end{aligned}
$$

Then, we use $\psi_{x}=(\log \psi)_{x} \psi$, define a wave number $k=(\log \psi)_{x}$, and obtain the PF equation in the form

$$
\tau \partial_{t} \phi=\eta^{2} \partial_{x x} \phi-f_{\phi}(\phi)+\left(2 \eta k-e_{0}\right) \eta \partial_{x} \phi,
$$

which recovers the PF equation (42) with $e=\left(2 \eta k-e_{0}\right)$.

Now, we solve the forward scattering problem. The first Lax equation (28) after substituting the operators and assuming $\lambda=\tau \eta k$ becomes

$$
\partial_{x} \psi+\phi \psi / \eta=k \psi
$$

Assuming the asymptotic behaviour $\phi \rightarrow 0$ at $x \rightarrow$ $-\infty$, we get

$$
\partial_{x} \psi=k \psi
$$

The solution of this equation reads

$$
\psi=\psi_{0}(k, t) e^{k x}
$$

where the amplitude $\psi_{0}(k, t)$ is a function of wave number and time.

Substituting $\psi$ in the second Lax equation (29), we obtain

$$
\partial_{t} \psi=\frac{\eta^{2}}{\tau} \partial_{x x} \psi+\frac{V(x)}{\tau \eta} \psi+\frac{e_{0}}{\tau} \phi \psi
$$

This equation describes the time evolution of the scattering amplitude $\psi_{0}(k, t)$. Using the asymptotic $V(x) \rightarrow 0$ at $x \rightarrow-\infty$, we obtain

$$
\partial_{t} \psi_{0}(k, t)=\frac{\eta^{2}}{\tau} k^{2} \psi_{0}(k, t)
$$

and

$$
\psi_{0}(k, t)=\psi_{0}(k, 0) e^{\frac{k^{2} \eta^{2}}{\tau} t}
$$

Hence, the solution of the forward problem has the form

$$
\psi(x, t)=\psi_{0}(k, 0) e^{k x} e^{v t}
$$

where $\nu=\frac{k^{2} \eta^{2}}{\tau}$.

Using the solution of (56), we solve the inverse scattering problem. First, we define the function $F(x, t)$ as

$$
F(x, t)=F_{0} e^{k x} e^{2 v t}
$$

and search the solution of (31) in the form $K(x, y, t)=$ $M(x, t) e^{k y}$. After substitution, we get

$$
\begin{aligned}
& M(x, t) e^{k y}+F_{0} e^{k(x+y)} e^{2 v t} \\
& \quad+\int_{x}^{+\infty} M(x, t) e^{k z} F_{0} e^{k(y+z)+2 v t} \mathrm{~d} z=0,
\end{aligned}
$$

then

$$
\begin{aligned}
& M(x, t)+F_{0} e^{k x+2 v t} \\
& \quad+M(x, t) F_{0} e^{2 v t} \int_{-\infty}^{x} e^{2 k z} \mathrm{~d} z=0,
\end{aligned}
$$

and finally

$$
M(x, t)+F_{0} e^{k x+2 v t}+M(x, t) F_{0} e^{2 v t} \frac{e^{2 k x}}{2 k}=0 .
$$

Hence

$$
M(x, t)=\frac{-2 k F_{0} e^{k x+2 v t}}{2 k+F_{0} e^{2 k x+2 v t}}
$$

and from (30), we obtain

$$
\phi(x, t)=-2 k^{2} \operatorname{sech}^{2}(-k x-v t-\delta),
$$

where $\delta=1 / 2 \log \left(F_{0} / 2 k\right)$. This solution has the form of the known solution of $\mathrm{KdV}$ equation. The difference is in the dependency of $v$ on $k$, which is quadratic. The comparison with the solution of (45) gives $k=\frac{1}{\eta}, \nu=\frac{1}{\tau}, v=\frac{\eta}{\tau}$, $e=1, e_{0}=1$. The functions are compared in Figure 2 for $\eta=\sqrt{2}$ and $t=0$.

\subsubsection{Variant II}

The second variant of the Lax operators reads

$$
\mathcal{L}=\tau \eta \partial_{x}+\tau \phi
$$

$$
\mathcal{A}=\frac{\eta e}{\tau} \partial_{x}+\frac{V(x)}{\tau \eta}-\frac{\eta}{\tau} \partial_{x} \phi
$$




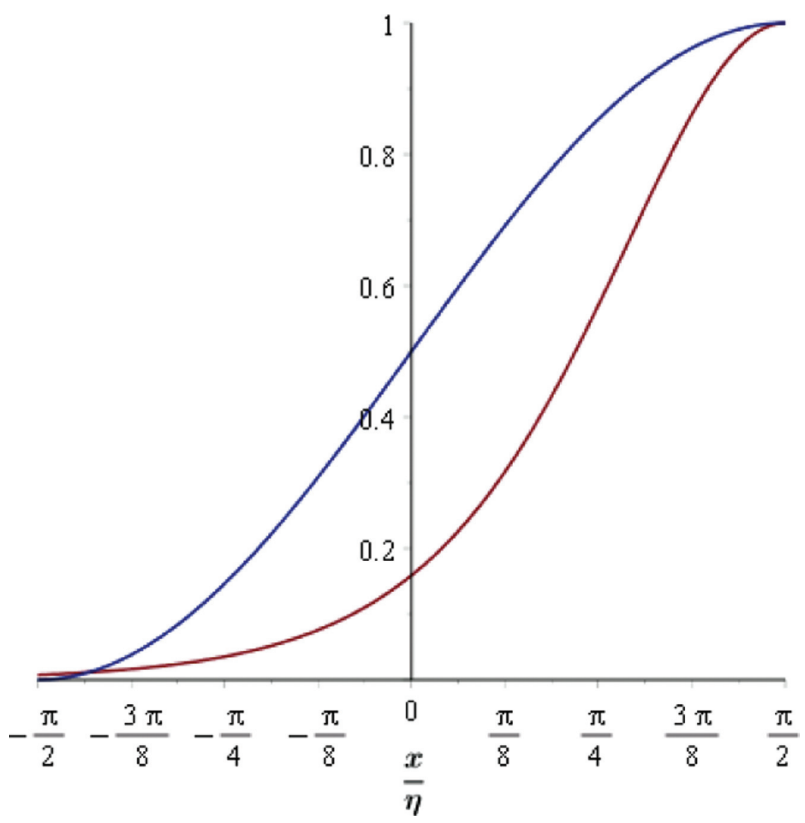

Figure 2: Comparison of the functions $\frac{1}{2} \sin \left(\frac{x}{\eta}\right)+\frac{1}{2}$ (top, blue) and $2 k^{2} \operatorname{sech}^{2}\left(-k x-\frac{\pi}{2}\right)$ (bottom, red) for $k=\frac{1}{\sqrt{2}}$ and $\eta=\sqrt{2}$.

The non-zero components of the Lax equation are

$$
\begin{aligned}
{\left[\partial_{x}, V(x)\right] \psi } & =f_{\phi}(\phi) \psi ; \\
{\left[\phi, \partial_{x}\right] \psi } & =\phi \psi_{x}-\phi_{x} \psi-\phi \psi_{x}=-\phi_{x} \psi ; \\
{\left[\partial_{x}, \phi_{x}\right] \psi } & =-\phi_{x x} \psi .
\end{aligned}
$$

After substitution in (27), we recover the PF equation (42) with arbitrary $e$ :

$$
\tau \partial_{t} \phi=\eta^{2} \partial_{x x} \phi-f_{\phi}(\phi)+\eta e \partial_{x} \phi .
$$

The first Lax equation has the same form as (50):

$$
\eta \partial_{x} \psi=-\phi \psi+k \eta \psi
$$

with the solution (52). The second Lax equation reads

$$
\partial_{t} \psi=\frac{\eta e}{\tau} \partial_{x} \psi+\frac{V(x)}{\tau \eta} \psi-\frac{\eta}{\tau}\left(\partial_{x} \phi\right) \psi
$$

Using the asymptotic $V(x) \rightarrow 0$ and $\phi_{x} \rightarrow 0$ at $x \rightarrow$ $-\infty$, we obtain

$$
\psi_{0}(k, t)_{t}=\frac{\eta e}{\tau} k \psi_{0}(k, t),
$$

which has the solution

$$
\psi_{0}(k, t)=\psi_{0}(k, 0) e^{\frac{k \eta}{\tau} t} .
$$

Hence, the solution of forward problem in the second variant is

$$
\psi(x, t)=\psi_{0}(k, 0) e^{k x} e^{\frac{k \eta n}{\tau} t} .
$$

Here, we obtain a linear dispersion relation, i.e. the frequency is proportional to $k, v=\frac{k \eta e}{\tau}$.

The procedure of the solution of the inverse scattering problem is similar to variant I. Finally, the solution is

$$
\phi(x, t)=-2 k \operatorname{sech}^{2}(-k x-v t-\delta) .
$$

Comparison with the sin-solution (45) gives $k=\frac{1}{\eta}$, $\nu=\frac{e}{\tau}$, and $v=\frac{v}{k}=\frac{\eta e}{\tau}$.

It can be shown that (67) is the solution of PF equation (42) with the potential $\tilde{f}(\phi)=2 \phi^{2}(1-\phi)$. It is also interesting to mention here that with this potential, the $\mathrm{PF}$ equation can be easily transformed to the KdV equation by taking the space derivatives of the two first terms on the right-hand side of the PF equation. This kind of potential is not stable, because it is unbounded from below and offers an infinite energy for a very large $\phi$. To overcome this difficulty, we suggest using the absolute value $|1-\phi|$, which produces the second minimum and allows to get a stable soliton. Figure 3 shows the moving soliton solved numerically by the PF equation (42) with $\tilde{f}(\phi)=2 \phi^{2}|1-\phi|$, the derivative of which is

$$
\tilde{f}_{\phi}(\phi)=2 \phi\left[2|\phi-1|+\frac{\phi(\phi-1)}{|\phi-1|}\right] .
$$

The parameters of the model are $\eta=20, e=1$, $\tau=0.5, \Delta t=1, \Delta x=1$. The numerical solution in Figure 3 sketches the analytical traveling wave solution

$$
\phi(x, t)=\operatorname{sech}^{2}\left(-\frac{x}{\eta}-\frac{t}{\tau}\right) .
$$

Note that the solution of (69) defines a symmetric soliton. Such kind of solitons are typically derived from a KdV type of equation, which is third order in the space derivative, while (69) is derived from a second-order PF equation.

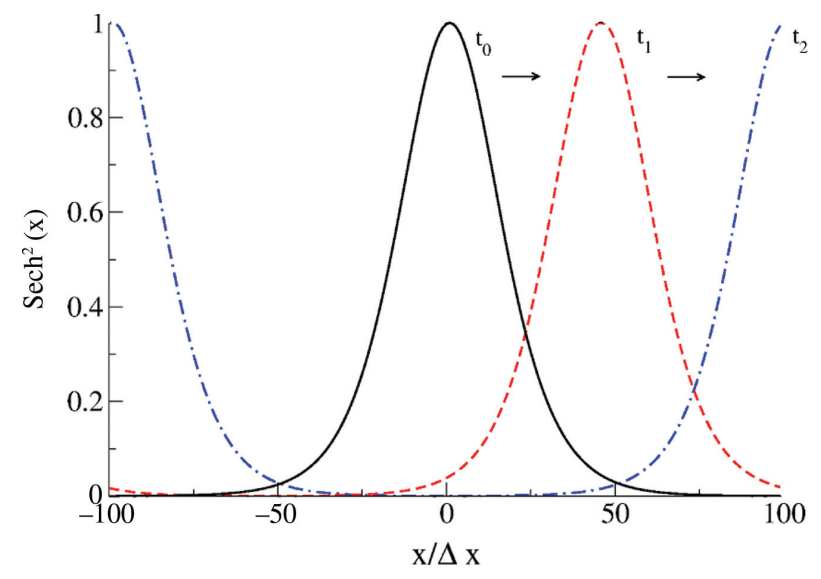

Figure 3: Traveling wave solution for the functions $\operatorname{sech}^{2}(-x / \eta-t / \tau)$ with periodic boundary conditions. 
The important difference in the type of soliton solutions, symmetric or antisymmetric, thereby seems to lie in the parity asymmetry of one operator, either the differential operator or the potential. Here, more future work is necessary. The form (68) opens a new class of symmetric soliton solutions.

\subsubsection{Variant III}

The third variant of the Lax operators is

$$
\begin{aligned}
& \mathcal{L}=\tau \eta \partial_{x}+\tau \phi, \\
& \mathcal{A}=\frac{\eta e}{\tau} \partial_{x}+\frac{1}{\tau \eta} V(x)-\frac{\eta}{\tau} \partial_{x} \phi+\frac{\gamma}{\tau},
\end{aligned}
$$

where $\gamma$ is added to variant II for the normalisation of the scattering data (see [25]).

Now, we consider the imaginary forms of Lax equations. Substituting the operators and taking $\lambda=\tau \eta k$, we get the first equation:

$$
i \partial_{x} \psi+i \phi \psi / \eta=k \psi \text {. }
$$

Assuming an asymptotic behaviour $\phi \rightarrow 0$ at $x \rightarrow \infty$, we obtain

$$
i \partial_{x} \psi=k \psi
$$

with the solution

$$
\begin{aligned}
& \psi=e^{-i k x}+b(k, t) e^{i k x} \text { for } x \rightarrow \infty, \\
& \psi=a(k, t) e^{-i k x} \text { for } x \rightarrow-\infty .
\end{aligned}
$$

This solution describes scattering from the right of the incident wave $e^{-i k x}$ on the potential $\phi, b(k, t)$ represents a reflection coefficient, and $a(k, t)$ is a transmission coefficient. The second Lax equation with the same asymptotic becomes

$$
i \partial_{t} \psi=\frac{\eta e}{\tau} \partial_{x} \psi+\gamma \psi
$$

By substitution of $\gamma=i k \eta e$ and $a(k, 0)=0$, we obtain

$$
b(k, t)=b(k, 0) e^{\frac{i 2 k \eta e}{\tau} t} .
$$

Hence, the solution of the forward problem has the form

$$
\psi(x, t)=b(k, 0) e^{-i k x} e^{2 i v t},
$$

where $\nu=\frac{k \eta e}{\tau}$.
To solve the inverse scattering problem, we define $F(x, t)$ as follows:

$$
F(x, t)=F_{0} e^{-i(k x-2 v t)} .
$$

We search for a solution in the form

$$
K(x, y, t) \sim M(x, t) e^{-i k y} .
$$

Substituting it in (31) results in

$$
\begin{aligned}
& M(x, t) e^{-i k y}+i F_{0} e^{-i k(x+y)} e^{2 i v t} \\
& +i \int_{x}^{+\infty} M(x, t) e^{-i k z} F_{0} e^{-i(k(y+z)-2 v t)} \mathrm{d} z=0, \\
& M(x, t)+F_{0} e^{-i(k x-2 v t)} \\
& \quad+M(x, t) F_{0} e^{i v t} \int_{x}^{+\infty} e^{-2 i k z} \mathrm{~d} z=0 .
\end{aligned}
$$

The last term cancels by adding the complex conjugate (c. c.) function. Hence,

$$
\begin{aligned}
M(x, t) & =-F_{0} e^{-i(k x-2 v t)}, \\
K(x, x, t) & =-F_{0} e^{-i(2 k x-2 v t)} .
\end{aligned}
$$

As a result, we obtain from (30):

$$
\phi(x, t)=-\frac{2 F_{0} k}{i} e^{-i(2 k x-2 v t)}=-\frac{F_{0}}{i \eta} e^{-i \frac{(x-v t)}{\eta}},
$$

where

$$
k=\frac{1}{2 \eta}, \nu=\frac{e}{2 \tau}, v=\frac{\nu}{k}=\frac{\eta e}{\tau} .
$$

By adding the c. c. function and normalising, a real solution can be found in the form of (45).

\subsection{Lax Pair for the Relativistic Case}

The PF equation for the relativistic singularity can be written as

$$
\tau \partial_{t} \phi=\eta^{2}\left(\partial_{x x} \phi-\frac{1}{c^{2}} \partial_{t t} \phi\right)+f_{\phi}(\phi)+\eta e \partial_{x} \phi .
$$

This equation has the form of the Klein-Gordon equation with advection. Using the solution that the singularity moves with the velocity $v=\eta e / \tau$ according to advection equation, we can replace $\phi_{t t}$ by $v^{2} \phi_{x x}$ [11]. Then, the equation becomes

$$
\tau \partial_{t} \phi=\eta_{v}^{2} \partial_{x x} \phi+f_{\phi}(\phi)+\eta e \partial_{x} \phi,
$$

where $\eta_{v}=\eta \sqrt{1-v^{2} / c^{2}}$. 
The Lax pair can be chosen based on the second variant (59)

$$
\begin{aligned}
\mathcal{L} & =\tau \eta_{v} \partial_{x}+\tau \phi, \\
\mathcal{A} & =\frac{\eta e}{\tau} \partial_{x}+\frac{1}{\tau \eta_{v}} V(x)-\frac{\eta_{v}}{\tau} \phi_{x} .
\end{aligned}
$$

With this choice, the equations for the pilot-wave function become

$$
\begin{gathered}
\eta_{v} \partial_{x} \psi_{x}=-\phi \psi+\psi, \\
\tau \partial_{t} \psi=\eta e \psi_{x}+\frac{V(x)}{\eta_{v}} \psi-\eta_{v} \partial_{x} \phi \psi
\end{gathered}
$$

\section{Quantum-PF Concept}

Let $\phi$ be a scalar quantum field that acts on a quantum state $\psi=a e^{-i \theta}$ of a vacuum oscillation. The Hamiltonian of such a system, which formally corresponds to a free energy of a closed thermodynamic system, can be expanded in the non-relativistic case (for the general case, see [11]):

$$
\begin{array}{r}
H=U_{0} \int_{\Omega}\left\langle\psi^{\dagger}|\hat{h}| \psi\right\rangle \mathrm{d} x, \\
\hat{h}(\phi)=\frac{\eta^{2}}{2}\left(\partial_{x} \phi\right)^{2}+f(\phi)
\end{array}
$$

as the integral over the domain $\Omega$ of the free energy density, which is defined as the expectation value of the nonlinear soliton operator $\hat{h}(\phi)$ acting on the pilot wave $\psi$. The first term can be calculated as

$$
\begin{aligned}
\left\langle\psi^{\dagger}\left|\partial_{x} \phi \partial_{x} \phi\right| \psi\right\rangle & \\
= & \partial_{x}\left(\phi \psi^{\dagger}\right) \partial_{x}(\phi \psi) \\
= & \left(\psi^{\dagger} \partial_{x} \phi+\phi \partial_{x} \psi^{\dagger}\right)\left(\psi \partial_{x} \phi+\phi \partial_{x} \psi\right) \\
= & \psi \psi^{\dagger}\left(\partial_{x} \phi\right)^{2}+\phi^{2} \partial_{x} \psi \partial_{x} \psi^{\dagger} \\
& +\phi \partial_{x} \phi\left(\psi \partial_{x} \psi^{\dagger}+\psi^{\dagger} \partial_{x} \psi\right) .
\end{aligned}
$$

The integration of the last term gives 0 . Then, by the relaxation dynamics

$$
\psi \psi^{\dagger} \tau \partial_{t} \phi=-\frac{1}{U_{0}} \frac{\delta H}{\delta \phi},
$$

we obtain

$$
\begin{aligned}
\psi \psi^{\dagger} \tau \partial_{t} \phi= & \eta^{2} \psi \psi^{\dagger} \partial_{x x} \phi-\eta^{2} \phi \partial_{x} \psi^{\dagger} \partial_{x} \psi \\
& +\psi \psi^{\dagger} f_{\phi}(\phi) .
\end{aligned}
$$

Substituting $\partial_{x} \psi^{\dagger} \partial_{x} \psi=\left(\theta_{x}\right)^{2} \psi \psi^{\dagger}$ and $f_{\phi}=\phi-\frac{1}{2}$ as defined in (44), we get the PF equation with the DO potential:

$$
\tau \partial_{t} \phi=\eta^{2} \partial_{x x} \phi+f_{\phi}-\eta^{2}\left(\theta_{x}\right)^{2} \phi,
$$

where $\eta^{2}\left(\theta_{x}\right)^{2}$ is a quantum driving force. Using the complex function $\phi=e^{-i \theta(x)}$ with $\theta_{x}=1 / \eta$, (91) can be rewritten as

$$
i \tau \partial_{t} \phi=\eta^{2} \partial_{x x} \phi+f_{\phi}+i \eta \partial_{x} \phi .
$$

Equation (90) has the similar meaning as (48), which is obtained by means of the Lax pair method. The function $\psi \psi^{\dagger}$ is an analogy of the function $\psi$ in Section 3. Hence, using the Lax method, we can find the Lax equations for $\psi$ and $\psi^{\dagger}$. For (92), we can choose the following Lax pair:

$$
\begin{aligned}
& \mathcal{L}=\tau \eta \partial_{x}+\tau \phi, \\
& \mathcal{A}=\frac{\eta^{2}}{\tau} \partial_{x x}+\frac{V(x)}{\tau \eta}+\frac{i}{\tau} \phi,
\end{aligned}
$$

where $V(x)=-\int f_{\phi}(\phi) \mathrm{d} x$. The non-zero components of the Lax equation are

$$
\begin{aligned}
{\left[\partial_{x}, V(x)\right] \psi } & =-f_{\phi}(\phi) \psi ; \\
{\left[\phi, \partial_{x x}\right] \psi } & =-\phi_{x x} \psi-2 \phi_{x} \partial_{x} \psi ; \\
{\left[\partial_{x}, \phi\right] \psi } & =\phi_{x} \psi .
\end{aligned}
$$

This is also valid for $\psi^{\dagger}$. Then, we can reproduce the $\mathrm{PF}$ equation (92) for the field $\phi$ by the Lax equation $i \mathcal{L}_{t}+$ $[\mathcal{L}, \mathcal{A}]=0$.

The corresponding Lax equation for the complex function $\psi\left(\right.$ or $\psi^{\dagger}$ ) have the form $i \psi_{t}=\mathcal{A} \psi$, i.e.

$$
i \tau \partial_{t} \psi=\eta^{2} \partial_{x x} \psi+\frac{V(x)}{\eta} \psi+i \phi \psi .
$$

An alternative method to obtain the evolution equation for a state function is the relaxation dynamics:

$$
\begin{aligned}
i \tau \partial_{t} \psi & =-\frac{1}{U_{0}} \frac{\delta H}{\delta \psi^{\dagger}} \\
& =\frac{\eta^{2}}{2} \phi^{2} \partial_{x x} \psi-\frac{\eta^{2}}{2}\left(\partial_{x} \phi\right)^{2} \psi-f(\phi) \psi .
\end{aligned}
$$

From this equation, we can see that the $\psi$ function exists only in the region where $\phi>0$ and it is bounded

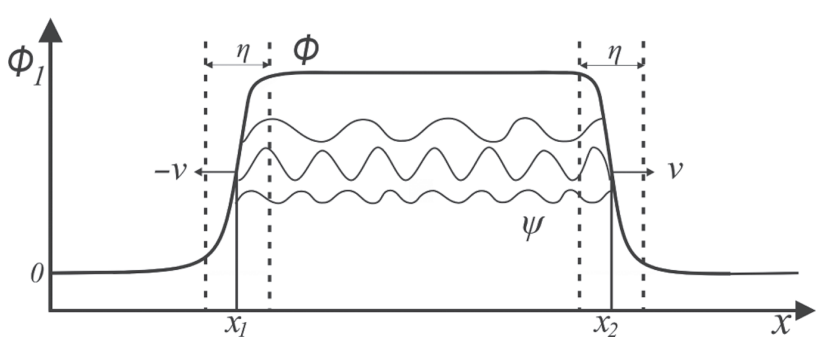

Figure 4: Doublon $\phi$ as a combination of a left- and a right-moving soliton defines the box for vacuum fluctuations $\psi$, which determine the spatial energy of the doublon. 
by the potential $f(\phi)$, which tends to 0 at $\phi \rightarrow 0,1$. Therefore, the problem is similar to the Schrödinger equation for a particle in a box potential (see below, Fig. 4).

\section{The Doublon in $1+1$ Dimensions}

Having the solution for the wave $\phi$ in the form of the socalled half-sided 'soliton' with spinor character regarding parity in space, we easily construct a double-sided 'soliton', which we will call 'doublon' as an antisymmetric pair of two half-sided solitons and the 'space' in between them:

$$
\phi= \begin{cases}\frac{1}{2}+\frac{1}{2} & \sin \left(\frac{\pi\left(x-x_{1}+v t\right)}{\eta}\right) \\ & \text { for }-\frac{\eta}{2} \leq x-x_{1}+v t<\frac{\eta}{2} \\ \frac{1}{2}-\frac{1}{2} & \sin \left(\frac{\pi\left(x-x_{2}-v t\right)}{\eta}\right) \\ & \text { for }-\frac{\eta}{2} \leq x-x_{2}-v t<\frac{\eta}{2} \\ 1 & \text { for } x_{1}-v t+\frac{\eta}{2} \leq x<x_{2}+v t-\frac{\eta}{2} \\ 0 & \text { otherwise. }\end{cases}
$$

The solution is depicted in Figure 4 in 1 dimension neglecting time. The doublon consists of an antisymmetric pair of two half-sided solitons and a finite space, attributed by to a 1-dimensional line coordinate $x$, where $\phi(x) \equiv 1$. This space is bounded by the non-local transition regions of width $\eta$ where $\frac{\partial}{\partial x} \phi(x) \neq 0$, the right- and left-moving soliton, which we identify with particles moving with relative velocity $v=v_{\mathrm{p}}$. Thereby, the doublon forms a 1dimensional box for vacuum fluctuations $\psi$. Regions outside the doublon, i.e. regions on the line coordinate $x$ with $\phi(x) \equiv 0$, have no physical meaning. The doublon forms the elementary building block of the physical world in the present concept. It unifies space-time and mass in a monistic structure. 'Mass', attributed by positive energy, is related to $\eta^{2}$, while space is attributed by negative energy $e$. The latter is easily calculated from quantum fluctuations with discrete spectrum $p$ and frequency $\omega_{p}=\frac{\pi c p}{2 \Omega}$, where $\Omega=\left|x_{1}-x_{2}\right|$ is the size of the doublon. According to Casimir [26], this has to be compared to a continuous spectrum. This yields the negative energy $e$ of space:

$$
e=\alpha \frac{h c}{4 \Omega}\left[\sum_{p=1}^{\infty} p-\int_{1}^{\infty} p \mathrm{~d} p\right]=-\alpha \frac{h c}{48 \Omega},
$$

where $\alpha$ is a positive, dimensionless coupling coefficient. We have used the Euler-MacLaurin formula in the limit $\varepsilon \rightarrow 0$ after renormalisation $p \rightarrow p e^{-\varepsilon p}$. The quantum fluctuations are the Schroedinger-type solutions of the $\psi$ wave in the $\mathrm{dBB}$ picture. Here, we have to note one important difference in the approaches (see also the discussions in Section 8). In the dBB program, the pilot wave is the primary object that lives in a given environment and drives the $u$ wave in the form of a soliton as the object of investigation. In the present concept, the $\phi$ wave in the form of the doublon defines the environment for the $\psi$ wave. Up to now, only the 'quasi-static' limit has been worked out, i.e. that the doublon solution is kept fixed for the quantum solution (98). The direct coupling of both waves by their phase in the transition region $0<\phi<1$ may be investigated using the Lax formalism. We leave a closer investigation of the coupling between the $\phi$ and $\psi$ waves and a dynamic coupled solution to future work.

\section{The Doublon Network in $1+1+2$ Dimensions}

To proceed towards a multi-dimensional (in the space coordinate) case, we have to

- define a set of doublons $\phi^{I}, I=1 \ldots N$ with a number $N>6$ for a 3-dimensional space filling network,

- define the interaction between doublons, and

- embed the description in 3+1-dimensional space time.

The second item will follow canonically from the postulate that the set of doublons is closed in itself, i.e.

$$
\sum_{I=1 \ldots N} \phi^{I}=1
$$

Using (87) for the free energy of an individual field $I$, $H \rightarrow H^{I}$, we define the total free energy $H=\sum_{I=1 \ldots N} H^{I}$. Then, conserving (99) (see [27] for details), we end up with the equation of motion for the doublons:

$$
\begin{aligned}
\tau \partial_{t} \phi^{I} & =-\frac{1}{N} \sum_{J=1 \ldots N}\left\{\frac{\delta}{\delta \phi^{I}}-\frac{\delta}{\delta \phi^{J}}\right\} \frac{H}{U_{0}} \\
& =-\frac{1}{N} \sum_{J=1 \ldots N} \Phi^{I J} .
\end{aligned}
$$

The last expression defines an antisymmetric object of dual character, the pair-exchange operator $\Phi^{I J}$ between two doublon fields $I$ and $J$. This beautiful result is a mere consequence of the system being closed in itself and leads to a natural decomposition of the multi-body 
interaction between the doublons into pair-wise contributions. Knowing the structure of doublons consisting of two antisymmetric solitons and the space between them, it is obvious that the interaction of doublons only happens in the soliton regions where $0<\phi^{I}<1$ with size $\eta$. This size is estimated in [11] to be $<10^{-15} \mathrm{~m}$, i.e. in the range of elementary particles in the classical sense. We will call this region 'quasi-local', meaning that we have a non-local theory with highly localised states. Within the quasi-local position of an elementary particle where all $N$ doublon fields may interact, the particle is understood as a junction between doublons. Although doublon fields are constructed along a 1-dimensional line coordinate, we may argue that the junctions form 0-dimensional object. Later, when introducing charges in Section 7, we will also discuss a finite width of the doublons to allow for optical excitations. Each pair-exchange operator $\Phi^{I J}$ carries an orientation from the half-sided soliton at the endpoint of the doublon $\phi^{I}$, considered as a spinor. Due to the isomorphism of spinors with the 3-dimensional SU(2) group, we therefore may embed the doublons into a 3-dimensional spatial environment within the quasi-local environment of a particle and a small surrounding. Here, we define the $3+1$-dimensional field variable $\tilde{\phi}^{I}(\vec{x}, t)$ for which a standard PF description in 3-dimensional space is applicable. Outside this local environment, the field collapses to the space like part of a doublon. We formally define the doublon as the trajectory, or path, of a classical flow between the endpoints of the doublon in space time. The advancing flow corresponding to doublon $I, \Xi_{A d v}^{I}$ and its time reversed equivalent, the retarded flow $\Xi_{\text {Ret }}^{I}$, connects space-time events $\left(\vec{x}_{1}, t_{1}\right)$ and $\left(\vec{x}_{2}, t_{2}\right)$. This path is determined from a minimum action condition $E\left(\tilde{\phi}^{I}\right)=0$ (see $[28,29])$ :

$$
\begin{aligned}
& \tilde{\phi}^{I}(\vec{x}, t)=\Xi_{A d v}^{I} \tilde{\phi}^{I}\left(\vec{x}_{1}, t_{1}\right) ; \\
& \tilde{\phi}^{I}(t, \vec{x})=\Xi_{R e t}^{I} \tilde{\phi}^{I}\left(\vec{x}_{2}, t_{2}\right) .
\end{aligned}
$$

The doublon then follows the probability $P$ to find non-zero values of the field $\tilde{\phi}^{I}(\vec{x}, t)$ at space point $\vec{x}$ and time $t$ in $3+1$ dimensions:

$$
P\left[\tilde{\phi}^{I}(\vec{x}, t), \tilde{\phi}^{I}\left(\vec{x}_{1}, t_{1}\right)\right]=\int \mathcal{D} \tilde{\phi}^{I} \delta_{D}\left[E\left(\tilde{\phi}^{I}\right)\right],
$$

where $\delta_{D}$ is the Dirac functional delta function.

Any event $(\vec{x}, t)$ laying of the doublon connecting the events $\left(\vec{x}_{1}, t_{1}\right)$ and $\left(\vec{x}_{2}, t_{2}\right)$ can be reached either via the action of advancing operator on $\left(\vec{x}_{1}, t_{1}\right)$ or equivalently the retarding operator on $\left(\vec{x}_{2}, t_{2}\right)$.

The doublon network is schematically depicted in Figure 6 for six interacting doublons forming a

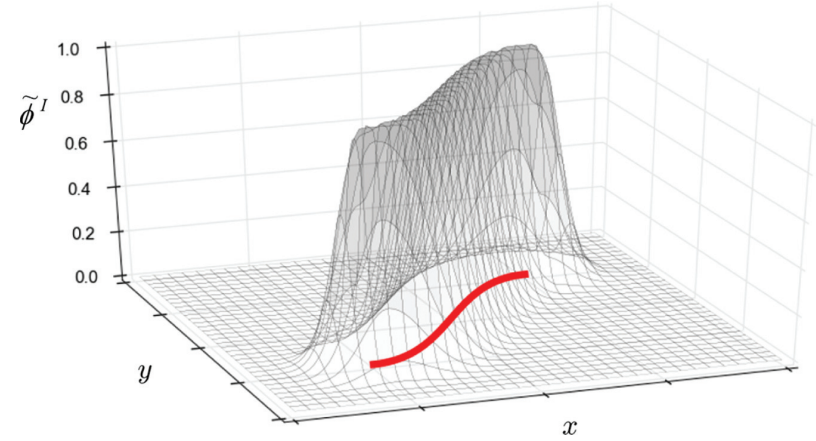

Figure 5: Sketch of a doublon represented by the $3+1$-dimensional field $\tilde{\phi}^{\prime}(\vec{x}, t)$.

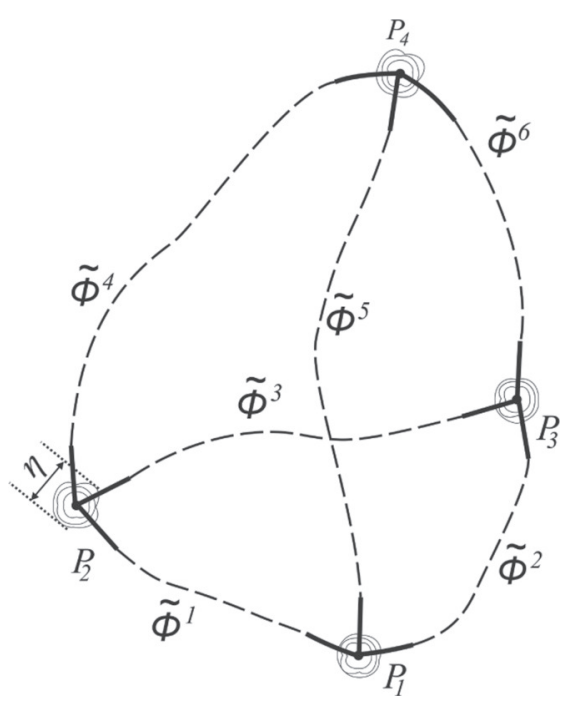

Figure 6: Doublon network for six interacting doublons $\tilde{\phi}^{1} \ldots \tilde{\phi}^{6}$ forming a 3-dimensional space filling environment with four particles $P_{1} \ldots P_{4}$. The junctions of size $\eta$ are stretched out by a minimum of three doublons $\phi^{\prime}$ defined on linear independent directions in 3-dimensional space.

3-dimensional space filling environment with four particles. As indicated, we define around the particles a 3-dimensional environment of size $\eta$, corresponding to the (inverse) gradient of the fields $\phi^{I}$, which interact in this quasi-local junction. As each doublon $\phi^{I}$ carries a different orientation in the 3-dimensional space it is embedded in, we define here the 3-dimensional field $\tilde{\phi}^{I}(\vec{x}, t)$. As indicated by the solid end-segments of the doublons, the volume of the junctions can uniquely be divided into volume segments assigned to the volumetric fields $\tilde{\phi}^{I}$. Outside the junctions, these volume fields collapse to tube-like objects along the definition (102), as indicated by the dashed lines representing the space part of the doublon. 


\section{PF Equation in Electromagnetic Field}

We will proceed here in the canonical way along the Ginzburg-Landau free energy functional for a superfluid phase defined by a doublon $\tilde{\phi}^{I}$ in electromagnetic field. The energy functional (87) now reads:

$$
H^{I}=U_{0} \int_{\Omega} h^{I}\left(\tilde{\phi}^{I}, \mathbf{A}\right) \mathrm{d} \mathbf{x},
$$

where

$$
\begin{aligned}
h^{I}\left(\phi^{I}, \mathbf{A}\right)= & \frac{1}{2}\left[\eta^{2} n_{s}\left|\left(\nabla-\frac{q \mathbf{A}}{\hbar}\right) \tilde{\phi}^{I}\right|^{2}\right. \\
& \left.+n_{s}\left|\tilde{\phi}^{I}\left(1-\tilde{\phi}^{I}\right)\right|+\frac{1}{4} \frac{\left(\nabla n_{s}\right)^{2}}{n_{s}}\left(\tilde{\phi}^{I}\right)^{2}+\frac{B^{2}}{\mu U_{0}}\right] .
\end{aligned}
$$

Here, $\mathbf{A}$ is the vector potential, $B$ is the magnetic field, $\mu$ is the magnetic permeability, $\mathbf{x}$ is the space vector, $q$ is the charge, $U_{0}=m c^{2}$ is the energy of a superfluid, $n_{s}=\psi \psi^{\dagger}$ is the concentration of a superfluid, $\eta=\frac{\hbar}{m c}$ is the interface width, and $\frac{B^{2}}{2 \mu}$ is the energy density of the magnetic field.

Using relaxation dynamic (89), we obtain the evolution equation for a phase in an electromagnetic field:

$$
\begin{aligned}
\tau \partial_{t} \tilde{\phi}^{I}= & \eta^{2}\left(\nabla-\frac{q \mathbf{A}}{\hbar}\right)^{2} \tilde{\phi}^{I}+\left(\tilde{\phi}^{I}-\frac{1}{2}\right) \\
& +\frac{1}{4} \frac{\left(\nabla n_{s}\right)^{2}}{n_{s}^{2}} \tilde{\phi}^{I} .
\end{aligned}
$$

In order to derive the kinetic equation for the superfluid, we minimise the functional $H^{I}$ with respect to A. First, we rewrite functional to collect all terms depending on $\mathbf{A}$ as

$$
\begin{aligned}
U_{0} h^{I}\left(\tilde{\phi}^{I}, \mathbf{A}\right)= & U_{0} h_{0}^{I}\left(\tilde{\phi}^{I}\right)-\frac{\hbar q n_{s}}{m} \mathbf{A} \tilde{\phi}^{I} \nabla \tilde{\phi}^{I} \\
& +\frac{q^{2} n_{s} A^{2}}{2 m}\left(\tilde{\phi}^{I}\right)^{2}-\frac{B^{2}}{2 \mu} .
\end{aligned}
$$

By substitution $\nabla \times \mathbf{A}=\mathbf{B}$, the variation of the last term with respect to $\mathbf{A}$ gives

$$
\begin{aligned}
\delta B^{2} & =\delta|\nabla \times \mathbf{A}|^{2}=\frac{\partial|\nabla \times \mathbf{A}|^{2}}{\partial(\nabla \times \mathbf{A})} \delta(\nabla \times \mathbf{A}) \\
& =2(\nabla \times \mathbf{A}) \delta(\nabla \times \mathbf{A}) \\
& =-2 \nabla((\nabla \times \mathbf{A}) \times \delta \mathbf{A})+2(\nabla \times \nabla \times \mathbf{A}) \delta \mathbf{A},
\end{aligned}
$$

where the integral of the first term is 0 by Gauss' theorem.
Finally, the minimisation of $H^{I}$ gives

$$
\frac{\delta H^{I}}{\delta \mathbf{A}}=-\frac{\hbar q n_{s}}{m} \tilde{\phi}^{I} \nabla \tilde{\phi}^{I}+\frac{q^{2} n_{s} \mathbf{A}}{m} \tilde{\phi}_{I}^{2}+\frac{\nabla \times \mathbf{B}}{\mu}=0 .
$$

Substituting $\nabla \times \mathbf{B}=\mu \mathbf{j}_{s}$, we obtain the second London equation:

$$
\mathbf{j}_{s}=-\frac{q^{2} n_{s}}{m} \mathbf{A}
$$

for the case of the uniform $\tilde{\phi}^{I}=1$.

\section{Discussion and Conclusion}

The $\mathrm{dBB}$ double-solution program sets a framework of coupled wave equations to represent 'particles' that are guided by a probability wave function in order to be consistent with observations of the quantum statistical behaviour of these particles. Let us recall the famous double-slit experiment: particles (photons or electrons or even fullerenes [30]) emitted on one side of a double slit form an interference pattern at the absorber screen. It must be stated clearly that an individual particle has only one strike on the absorber screen. The interference pattern, however, also appears if the objects are recorded with some time delay, i.e. that the events can be treated uncorrelated. The situation is depicted in Figure $7 \mathrm{a}$ and b. An individual particle only has one strike at the absorber screen, while a number of particles form the interference pattern. In the $\mathrm{dBB}$ picture, the pilot wave replaces the probability wave in the Copenhagen interpretation. From a rational point of view, these waves can be seen as kind of a Fourier-transformed snapshot of the experimental setup. The time needed for transport is not included in this interpretation. Also, the scale on which interference is observed only depends on the relation between the distance of the slits and the characteristic wavelength of the pilot wave, i.e. the dominating Fourier component. This solution is a classical wave solution negating the existence of discrete objects, the particles. It can be obtained by solving the differential wave-mechanical equation for given boundary conditions, which represent the environment: emitter, absorber screen, and double-slit barrier. Thus, it is not astonishing that the slit experiment can be repeated with water waves and small cork particles in the classroom, or in a more sophisticated setup with oil droplets in the laboratory [31, 32]. Compare also the hydrodynamic interpretation of quantum mechanics by Madelung in the 1920s [33] and see [M. Hatifi, R. Willox, S. Colin and T. Durt, Entropy 20, 780-32(2018)] for further details. 
a

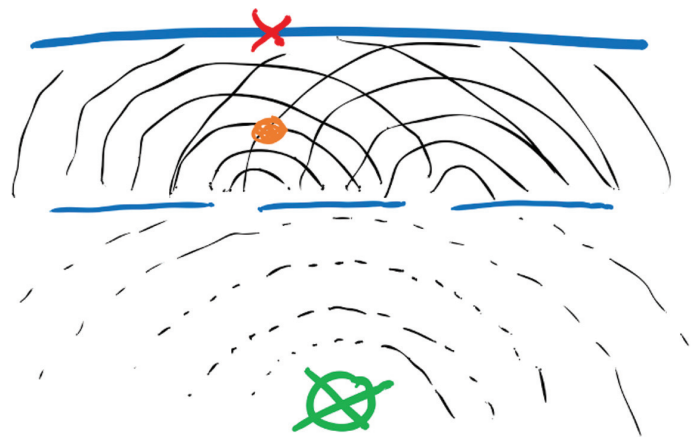

b

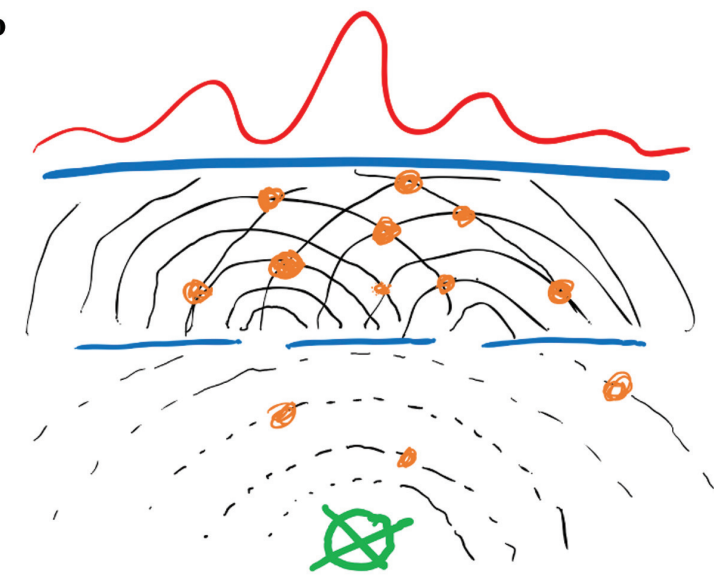

c

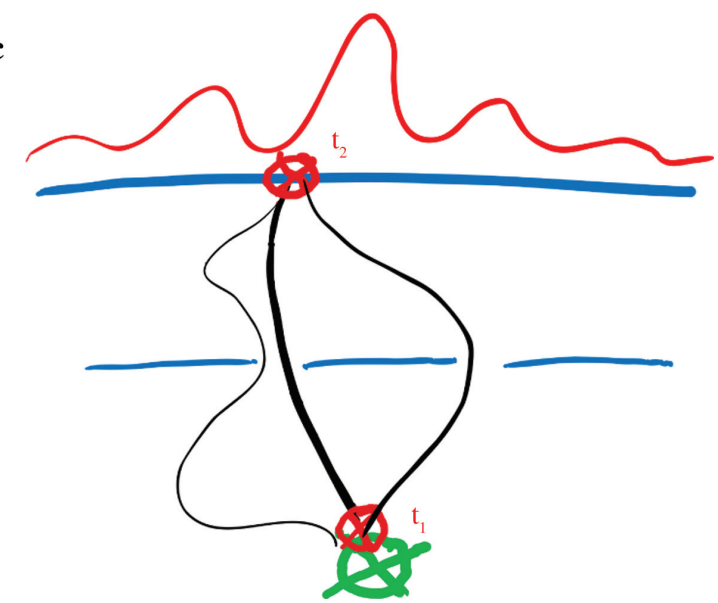

Figure 7: Double-slit experiment: (a) probability wave with one individual particle, (b) probability wave with many particles, and (c) path integral for a single particle trajectory.

Now, we must recall one major criticism of Einstein (and others) on these interpretations of quantum mechanics: that they anticipate an instantaneous knowledge of the situation in the experiment. The time needed for transport is not considered. An elegant solution to this problem is given by Feynman's path integral theory [34] (Fig. 7c). The probability to detect a particle at a special position at the absorber screen is determined by the action of the particle along different paths. A global wave within the whole experimental setup does not exist and the time of transport is consistently considered. Also, the scale on which quantum interference can be observed is determined by the action in comparison to Planck's quantum. Thus, one can clearly distinguish between quantum effects and classical hydrodynamic effects [31, 32].

The path integral picture leads us directly to the doublon-network model in Section 6, where the doublon represents a kind of elementary space quantum connecting emitter and absorber. The doublon connects these points by a flow of quantum fluctuations. Elementary particles by themselves are defined from gradient contributions, half-sided solitons, on both ends of the doublon. The concept is consistent with the approach of dBB but distinct in several aspects, the most important of which is the topology of space and the definition of particles.

As we can see from Section 2, the PF formalism allows to treat both forms of the particle representation: the symmetric solution and the half-sided solution (Fig. 1). Due to their parity, one may identify the symmetric solution with a bosonic particle and the antisymmetric, or half-sided, solution with a fermionic particle. We state that fermionic particles always have to come in pairs and that there is a conservation constraint. For bosonic particles, however, no conservation is expected as their creation or annihilation does not change the global wave solution apart from the individual position of the bosons. We may speculate that the space doublons $\tilde{\psi}^{I}$ possess a transverse width that may be determined by a bosonic wave solution in transverse direction according to (68) and (69). In this transverse direction, optical excitations are possible corresponding to electromagnetic waves. More future work is needed to investigate such solutions.

In Section 2, we have considered several variants of $\mathrm{PF}$ equations within the framework of the $\mathrm{dBB}$ doublesolution program. Special equations are found that define particle-like wave solutions. More technically, we have separated the real parts of the PF equation, which are responsible for the phase evolution, and the imaginary parts for the amplitude evolution. A promising variant seems to be the definition of the particle velocity as the velocity of an amplitude of a superwave whose phase is the probability wave function (see Section 2.2). Both the amplitude and the phase are solutions of Schrödingertype equations, which have the form of the transformed $\mathrm{PF}$ equation. Hence, the particle is defined as a wave function that is coupled to the probability wave function through the superwave. If the state function changes due to a quantum or other forces, then the particle will change its velocity, which depends on the phase of the probability 
function, whereas the quantum force is defined by the amplitude of the probability function. Therefore, the probability function works as the pilot wave in analogy the $\mathrm{dBB}$ theory.

Finally, we have demonstrated the potential of the Lax formalism with the given explicit forms of the Lax operators to trace the coupling of the particle wave $\tilde{\psi}$ and the probability wave $\phi$ in Section 2, as well as the coupling of the quantum oscillations $\tilde{\psi}$ and the doublon $\phi^{I}$ in Section 4.

In conclusion, our findings provide a new interpretation of particles in quantum mechanics and new opportunities for the formulation of the quantum wave equations. Our treatment goes significantly beyond the original dBB program by establishing a consistent set of wave equations derived by variational principles. Further, it allows predictions (see [11]) regarding structure formation in the universe and its accelerating expansion [35].

Acknowledgements: The authors would like to thank Fathollah Varnik (Bochum) for support with discussions and suggestions, and Dmitry Medvedev (Bochum/ Novosibirsk) for critical reading of the manuscript.

\section{References}

[1] D. Bohm, Phys. Rev. 85, 166 (1952).

[2] D. Bohm, Phys. Rev. 85, 180 (1952).

[3] L. de Broglie, Nonlinear Wave Mechanics: A Causal Interpretation, Elsevier, Amsterdam 1960.

[4] L. de Broglie, Proc. Znt. Sch. Phys. Enrico Fermi 49, 346 (1971).

[5] H. D. Zeh, Found. Physics Lett. 12, 197 (1999).

[6] J. S. Bell, Physics 1, 195 (1964).

[7] S. Colin, T. Durt and R. Willox, Annales de la Fondation Louis de Broglie 42, 19 (2017).

[8] A. Constantin, Invent. Math. 166, 523 (2006).
[9] B. Zwiebach, A First Course in String Theory. 2nd ed., Cambridge University Press, Cambridge, UK 2009.

[10] E. Zaslow, in: The Princeton Companion to Mathematics (Ed. T. Gowers), Princeton University Press, Princeton, NJ, USA 2010.

[11] I. Steinbach, Z. Naturforsch. A, 72, 51 (2017; erratum to appear, 2020).

[12] W. J. Boettinger, J. A. Warren, C. Beckermann, and A. Karma, Annu. Rev. Mater. Res. 32, 163 (2002).

[13] I. Steinbach, Modell. Simul. Mater. Sci. Eng. 17, 073001 (2009).

[14] I. Steinbach, Annu. Rev. Mater. Res. 43, 89 (2013).

[15] D. Korteweg and G. de Vries, Philos. Mag. 5, 422 (1895).

[16] A. C. Scott, F. Y. F. Chu, and D. W. McLauchlin, Proc. IEEE 61, $1443,1973$.

[17] N. S. Manton, Nonlinearity 21, T221 (2008).

[18] L. D. Landau and E. M. Lifshitz, Statistical Physics Part 1, third revised edition 1980, Pergamon, Oxford 1959.

[19] W. Craig, Philos. Trans. R. Soc. Lond. A 360, 2127 (2002).

[20] C. S. Bohun and F. I. Cooperstock, Phys. Rev. A 60, 4291 (1999).

[21] S. O. Olsen, Acta Phys. Acad. Sci. Hung. 37, 97 (1974).

[22] T. Ihle and H. Müller-Krumbhaar, Phys. Rev. Lett. 70, 3083 (1993).

[23] P. Lax, Pure Applied Math. 21, 467 (1968).

[24] V. E. Zakharov and A. B. Shabat, Sou. Phys.-JETP 34, 62 (1972).

[25] S. P. Novikov, S. V. Manakov, L. P. Pitaevskii, and V. E. Zakharov, The Theory of Solitons: The Inverse Scattering Method, Consultants, New York 1984.

[26] H. Casimir, Proc. Kon. Nederland. Akad. Wetensch. B51, 793 (1948).

[27] I. Steinbach and F. Pezzolla, Physica D 134, 385 (1999).

[28] G. F. Mazenko, Phys. Rev. E 83, 041125 (2008).

[29] M. Bartelmann, F. Fabis, D. Berg, E. Kozlikin, R. Lilow, et al., New J. Phys. 18, 043020 (2016).

[30] S. Gerlich, S. Eibenberger, M. Tomandl, S. Nimmrichter, K. Hornberger, et al., Nat. Commun. 2, 263 (2011).

[31] Y. Couder, S. Protiere, E. Fort, and A. Boudaoud, Nature 8, 208 (2005).

[32] Y. Couder and E. Fort, Phys. Rev. Lett. 97, 15410 (2006).

[33] E. Madelung, Z. Phys. 40, 322 (1927).

[34] R. Feynman, Rev. Mod. Phys. 20, 367 (1948).

[35] A. G. Riess, A. V. Filippenko, P. Challis, A. Clocchiattia, A. Diercks, et al., Astronom. J. 116, 1009 (1998). 